\title{
Correlative 3D Characterization of High Temperature Oxide Scales on Co-Base Superalloys Using Nano-CT and FIB/SEM Tomography
}

\author{
Janis Wirth ${ }^{1 *}$, Malte Lenz ${ }^{1}$, Silvan Englisch ${ }^{1}$, Jan Rosiwal ${ }^{1}$, Nadine Buchinger ${ }^{1}$, Martin Weiser ${ }^{2}$, \\ Benjamin Apeleo Zubiri ${ }^{1}$, Sannakaisa Virtanen ${ }^{2}$ and Erdmann Spiecker ${ }^{1 *}$ \\ 1. Institute of Micro- and Nanostructure Research (IMN) \& Center for Nanoanalysis and Electron \\ Microscopy (CENEM), Friedrich-Alexander University Erlangen-Nuremberg, Germany. \\ 2. Chair for Surface Science and Corrosion, Friedrich-Alexander University Erlangen-Nuremberg, \\ Germany. \\ * Corresponding authors: janis.wirth@fau.de, erdmann.spiecker@fau.de
}

In this contribution, we report on two Co-base superalloys oxidized under synthetic air $\left(20 \% \mathrm{O}_{2}\right)$ at $900^{\circ} \mathrm{C}$ for $12-100 \mathrm{~h}$. A simple ternary superalloy (Co-9Al-9W) and a multinary superalloy (ERBOCo-1) are compared [1]. The superior oxidation properties of the multinary alloy have been shown in previous studies by thermogravimetry and the respective mass gain during isothermal oxidation [2]. To understand this improvement, we combined electron microscopy and X-ray nano-tomography (NanoCT) to reveal the three-dimensional (3D) structure and chemistry of the oxide scales including their layered structure, depth-dependent porosity, and formation of foreign phases. Cross-section images suggest that the ternary alloy Co-9Al-9W exhibits a highly porous transition layer and topologically closed packed (TCP) phase formation while the oxide scales of ERBOCo-1 consist of two porous layers, containing spinels and oxides, and a discontinuous alumina barrier layer.

Nano-CT allows non-destructive 3D investigations of materials across multiple length scales. The laboratory ZEISS Xradia 810 Ultra X-ray microscope uses Fresnel zone plate optics to achieve 3D resolutions down to $(50 \mathrm{~nm})^{3}$ and can be equipped with a Zernike phase ring enabling phase contrast in addition to standard absorption contrast microscopy [3]. Absorption contrast imaging is well suited to study sample regions with much increased density compared to the surrounding matrix. In the case of Co-base superalloys, this holds true for TCP-phases enriched in refractory elements like, e.g., W, Ta, and Re. Phase contrast imaging can be employed to study weakly absorbing materials and to discriminate between materials exhibiting similar densities. Thus individual pores and pore networks in the multilayer oxide scales can be nicely revealed. Due to this flexible use of imaging modes, Nano-CT is perfectly suited for correlative 3D studies of complex materials systems, especially in combination with a Dual-Beam Focused Ion Beam Scanning Electron Microscope (FIB/SEM), which can be employed for preparation of Nano-CT samples as well as for correlative tomography experiments.

We developed a specialized workflow (Figure 1), which enables the combination of structural 3D characterization with precise chemical analysis. Since Nano-CT is a non-destructive imaging technique, the same sample can be reused for further analyses. In order to discriminate between different elemental compositions, 3D-FIB/SEM tomography is combined with energy dispersive X-ray spectroscopy (EDXS). The 3D datasets of Nano-CT and subsequent 3D-FIB/SEM tomography with EDXS mappings of the same region are reconstructed and combined (Figure 1e). Utilizing the combined 3D volumes, a morphological and chemical analysis of individual pore structures and different phases is possible (Figure 2) [4]. 
References:

[1] M Weiser et al., Corr. Sci. 135 (2018), p. 78.

[2] N Volz et al., Met. Mat. Trans. A 49 (2018), p. 4099.

[3] A Tkachuk et al., Z. Kristallogr. 222 (2007), p. 650.

[4] The authors acknowledge the financial support of the DFG within the frameworks of the collaborative research center SFB/TR 103 (projects A7 and A5) and the project SP648/8 "Highresolution X-ray microscopy for correlative tomography, high throughput screening and in situ mechanical testing of structural and functional materials".

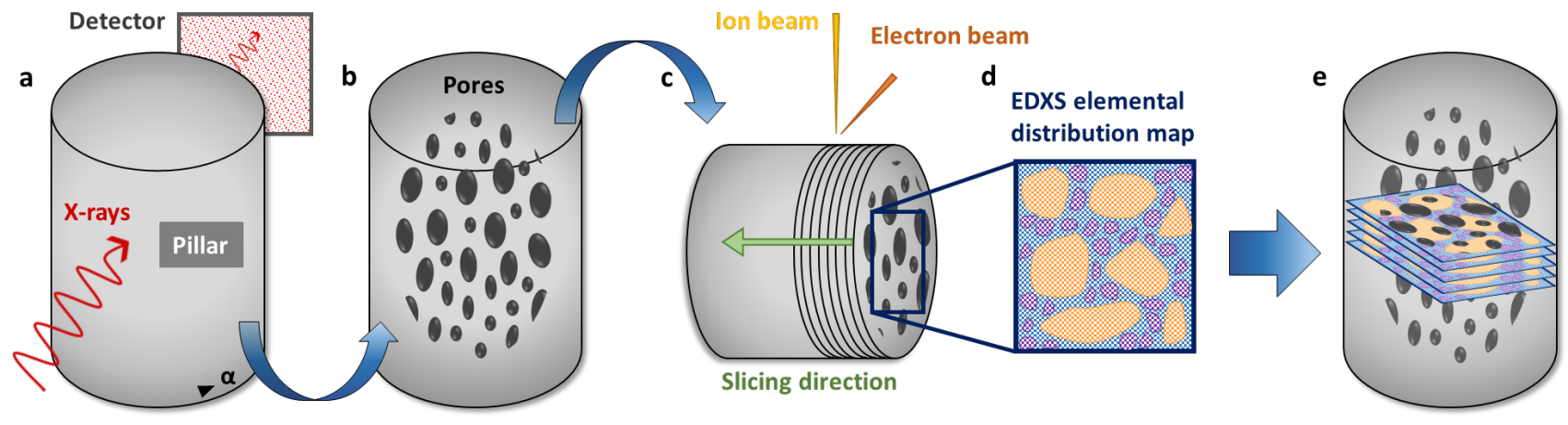

Figure 1. Workflow scheme of combined Nano-CT and 3D-FIB/SEM-EDXS tomography: (a) Nano$\mathrm{CT}$ tilt series acquisition in phase or absorption contrast. (b) Volume rendering of the 3D reconstruction reveals the pore structure and foreign phases inside the oxide scale. (c) 3D-FIB imaging in tandem with (d) EDXS mapping reveals the chemical composition of various phases in the 3D structure. (e) Merging of 3D-datasets enables discrimination between pore structures, matrix and aluminum oxide phases.
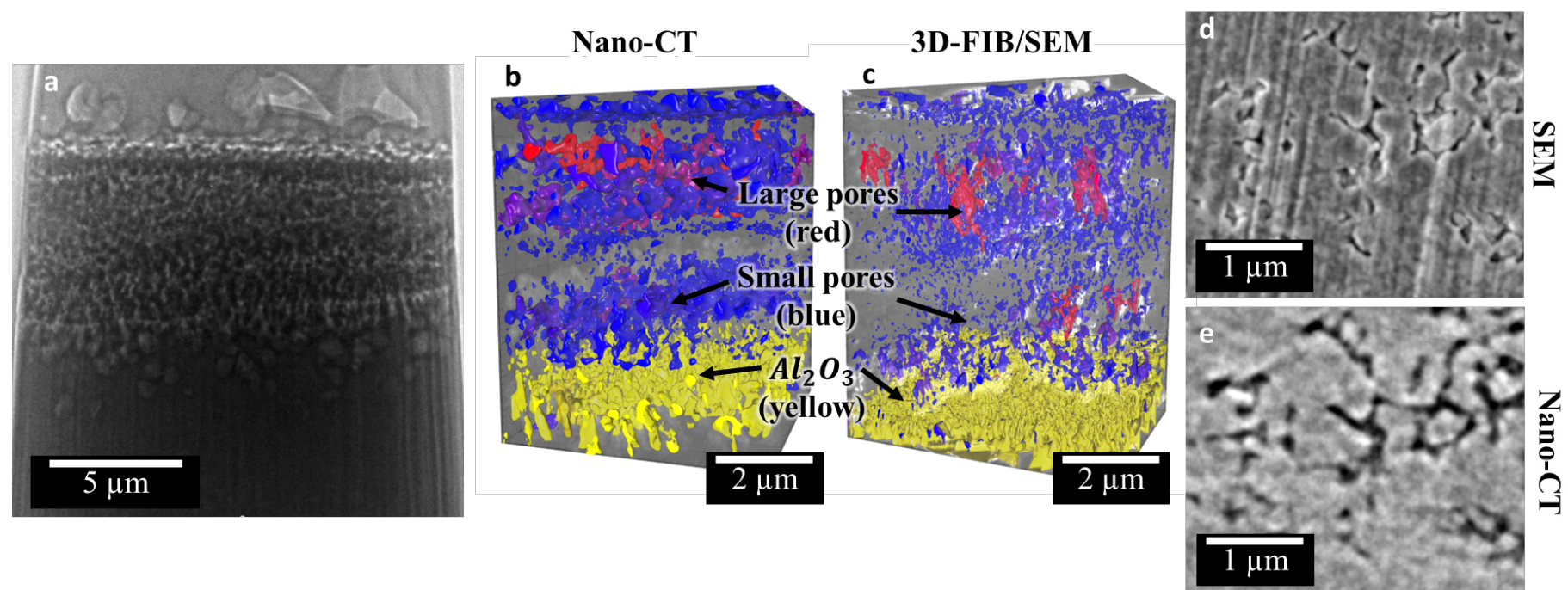

Figure 2. (a) Phase contrast X-ray microscopy image taken from Nano-CT tilt series of high temperature oxide scale in ERBOCO-1 sample. (b) 3D volume rendering of the reconstructed Nano-CT dataset revealing large pores $\left(>0.200 \mu \mathrm{m}^{3}\right.$ : red), small pores (blue) and aluminum oxide phases (yellow). (c) 3D-FIB/SEM tomography dataset taken from the same region studied by Nano-CT. Direct correlation between (d) one FIB/SEM slice image and (e) the corresponding virtual slice of the Nano-CT $3 \mathrm{D}$ volume allows precise thresholding and quantitative analysis of pore sizes in the oxide scale. 